\title{
Review Article \\ Chondroitin Sulfate Proteoglycans: Structure-Function Relationship with Implication in Neural Development and Brain Disorders
}

\author{
Speranta Avram, ${ }^{1}$ Sergey Shaposhnikov, ${ }^{2}$ Catalin Buiu, ${ }^{1,3}$ and Maria Mernea ${ }^{1}$ \\ ${ }^{1}$ Anatomy, Animal Physiology and Biophysics Department, Faculty of Biology, University of Bucharest, \\ 91-95th Independentei Street, 050095 Bucharest, Romania \\ ${ }^{2}$ Norgenotech AS, Totenvegen 2049, 2848 Skreia, Norway \\ ${ }^{3}$ Automatic Control and Systems Engineering Department, Faculty of Automatic Control and Computers, \\ "Politehnica" University of Bucharest, 313th Independentei Street, 060042 Bucharest, Romania
}

Correspondence should be addressed to Maria Mernea; maria.mernea@bio.unibuc.ro

Received 28 February 2014; Revised 28 April 2014; Accepted 28 April 2014; Published 14 May 2014

Academic Editor: Sun-On Chan

Copyright (C) 2014 Speranta Avram et al. This is an open access article distributed under the Creative Commons Attribution License, which permits unrestricted use, distribution, and reproduction in any medium, provided the original work is properly cited.

Chondroitin sulfate proteoglycans (CSPGs) are extracellular matrix components that contain two structural parts with distinct functions: a protein core and glycosaminoglycan (GAG) side chains. CSPGs are known to be involved in important cell processes like cell adhesion and growth, receptor binding, or cell migration. It is recognized that the presence of CSPGs is critical in neuronal growth mechanisms including axon guidance following injury of nervous system components such as spinal cord and brain. CSPGs are upregulated in the central nervous system after injury and participate in the inhibition of axon regeneration mainly through their GAG side chains. Recently, it was shown that some CSPGs members like aggrecan, versican, and neurocan were strongly involved in brain disorders like bipolar disorder (BD), schizophrenia, and ADHD. In this paper, we present the chemical structure-biological functions relationship of CSPGs, both in health state and in genetic disorders, addressing methods represented by genome-wide and crystallographic data as well as molecular modeling and quantitative structure-activity relationship.

\section{CSPGs Structure-Biological Activity Relationship in Health State}

Over the past decade many studies were focused on the complex interactions between neuron and glia [1-4] and the general opinions of these studies were that extracellular matrix molecules (ECM) like CSPGs are involved in the synapsis process among neurons and astrocytes.

The neural ECM include the CSPGs of the lectican family $[1,4]$ as well as glycoproteins such as the tenascins [5]. Lecticans, a family of hyaluronan binding proteoglycans, are represented by aggrecan (ACAN), versican (VCAN), neurocan (NCAN), and brevican (BCAN) $[4,6]$ and have an important role in neuronal growth mechanisms represented by neuroplasticity, axon guidance, or neuron repair processes following injury to the spinal cord or brain [4, 7-9]. It was shown that CSPGs levels fluctuate during the brain development process, as high values were detected in immature brain of embryos but not in the mature brain of normal adults $[6,10,11]$. During postnatal neural development, CSPGs play an active role in axon guidance, with the movement of axons being established through second messengers such as calcium and cyclic nucleotides [7].

Structurally, CSPGs contain a protein core covalently linked to one or more unbranched GAG chains that play different roles in CSPGs biological functions [12,13]. Several studies have emphasized the important roles of GAG chains in the growth-inhibitory and neuron regenerative processes $[1,12,14-16]$. Also, it was indicated that most functions of CSPGs are predominantly performed by the chondroitin sulfate moieties, while the core proteins act as a scaffold [12]. Even if it was supposed that in vivo the CSPG core proteins do not play a critical role, several very recent studies showed that CSPG core proteins inhibit neurite outgrowth 
TABLE 1: Chondroitin sulfate enzymes in human.

\begin{tabular}{|c|c|c|}
\hline Enzymes & Abbreviation & Chromosomal location \\
\hline \multicolumn{3}{|c|}{ Glycosyltransferases involved in synthesis of the tetrasaccharide linkage region } \\
\hline Xylosyltransferase & XylT & $16 \mathrm{p} 12.3$ \\
\hline$\beta 1,4-G a l a c t o s y l t r a n s f e r a s e-I$ & GalT-I & $5 q 35.2-q 35.3$ \\
\hline$\beta 1,3$-Galactosyltransferase-II & GalT-II & $1 \mathrm{p} 36.33$ \\
\hline$\beta 1,3$-Glucuronyltransferase-I & GlcAT-I & $11 \mathrm{q} 12.3$ \\
\hline \multicolumn{3}{|c|}{ Glycosyltransferases involved in synthesis of the repeating disaccharide region of CS chains } \\
\hline \multirow{3}{*}{ Chondroitin synthase } & ChSy-1 & $15 \mathrm{q} 26.3$ \\
\hline & ChSy-2 & $5 q 23.3$ \\
\hline & ChSy-3 & $7 \mathrm{q} 36.1$ \\
\hline Chondroitin polymerizing factor & $\mathrm{ChPF}$ & $2 q 35$ \\
\hline \multirow{2}{*}{ Chondroitin transferase } & ChGn-1 & $8 \mathrm{p} 21.3$ \\
\hline & ChGn-2 & $10 \mathrm{q} 11.21$ \\
\hline \multicolumn{3}{|c|}{ Sulfotransferases and epimerases } \\
\hline \multirow{3}{*}{ Chondroitin 4-O-sulfotransferase } & C4ST-1 & $12 q$ \\
\hline & C4ST-2 & $7 \mathrm{p} 22$ \\
\hline & C4ST-3 & $3 q 21.3$ \\
\hline Dermatan 4-O-sulfotransferase & D4ST-1 & $15 q 15.1$ \\
\hline Chondroitin 6-O-sulfotransferase & C6ST-1 & $10 \mathrm{q} 22.1$ \\
\hline Uronyl 2-O-sulfotransferase & UST & $6 \mathrm{q} 25.1$ \\
\hline GalNAc 4-sulfate 6-O-sulfotransferase & GalNAc4S-6ST & $10 \mathrm{q} 26$ \\
\hline \multirow{2}{*}{ Glucuronyl C-5 epimerase } & DS-epil & $6 \mathrm{q} 22$ \\
\hline & DS-epi2 & $18 \mathrm{q} 22.1$ \\
\hline
\end{tabular}

and changes in expression of these proteins genes lead to psychiatric disorders [17-19]. Iseki et al. [20] studied the functional roles of CSGPs protein core and they noticed the following: (i) CSGP type NG2 is a major inhibitor of axonal growth in vitro; (ii) VCAN isoform V2 inhibits neurite outgrowth in the central and peripheral neurons, while (iii) BCAN is an inhibitor of neurite outgrowth and (iv) BCAN and NCAN mRNAs are upregulated in astrocytes in central nervous system glial scars.

Biosynthesis and degradation process of CSPGs were studied in detail by Kitagawa et al. [21, 22], Laabs et al. [12], Izumikawa et al. [23, 24], and recently Mikami and Kitagawa [25]. In these studies it was shown that the biosynthesis of CSPGs is a very complex process that requires the presence of the entire enzymatic machinery, broadly distributed in tissues, including the brain [12,21-26]. Enzymes are represented by glycosyltransferases, glucuronyltransferase-I, chondroitin synthase family of enzymes, and chondroitin polymerizing factor [12, 21-26]. Some of chondroitin sulfate enzymes and their chromosomal location and abbreviation are presented in Table 1 [25]. The entire mechanism of CSGPs action in central nervous system is unknown, but several studies [20, 27] showed that, at axonal level, the removal of GAG chains by chondroitinase $\mathrm{ABC}$ allowed some axonal regrowth.

NCAN is a significant component of the ECM, and its level is modulated by many factors. Important details about the structural features of NCAN were presented in previous studies $[12,18,28-32]$ which described the complete coding sequence of the human NCAN mRNA as well as mapping data, expression analysis, and genomic structure. Investigation of NCAN gene conservation among several eukaryotic species [33] indicated a close homology between sequences of NCAN from Homo sapiens (human), Pan troglodytes (chimpanzee), Macaca mulatta (Rhesus monkey), Canis lupus (dog), Bos taurus (cattle), Mus musculus (house mouse), Rattus norvegicus (Norway rat), and Gallus gallus (chicken). The amino acid sequence of human NCAN shows $63 \%$ identity with both the mouse and the rat sequences [28].

Structural studies on NCAN $[12,18,28-30]$ revealed the following: (i) full-length NCAN core protein from human species comprises 1321 amino acids with a molecular weight of $220 \mathrm{kDa}$; (ii) NCAN gene is found on chromosome $19 \mathrm{p12}$; (iii) NCAN contains 5 to $6 \mathrm{~N}$-linked and up to $40 \mathrm{O}$ linked oligosaccharides; (iv) NCAN presents three active domains, namely, a N-terminal hyaluronan binding domain, a C-terminal lectin-like domain (CLD), and a central GAG attachment region that has no homology with other family members.

It has been shown that members of lectican family are characterized by similarity in N-terminal globular hyaluronan binding domains and CLDs but differ considerably in their central regions [35] which may induce different biological functions for lectican family members. The domain structure of ACAN, VCAN, NCAN, and BCAN is presented in Figure 1. 


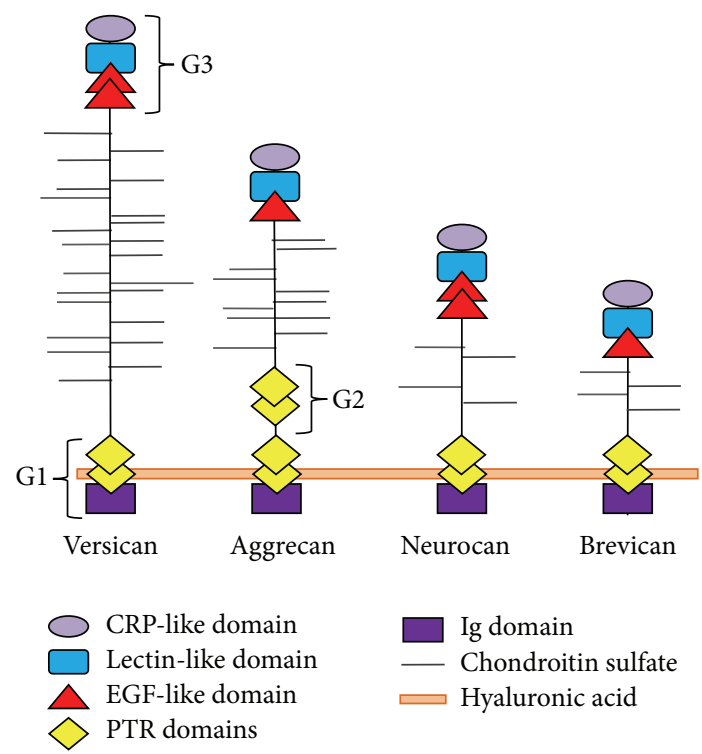

FIGURE 1: Domain structure of human VCAN, ACAN, NCAN, and BCAN. The schematic representation was performed based on information retrieved from UniProt database [34], entries P13611 (human VCAN sequence), P16112 (human ACAN sequence), O14594 (human NCAN sequence), and Q96GW7 (human BCAN sequence). The four CSPGs present the following: (i) an $\mathrm{N}$ terminal G1 domain comprising an immunoglobulin-like domain (Ig domain) and proteoglycan tandem repeats (PTR) domains involved in binding hyaluronic acid, (ii) a chondroitin sulfate attachment region, (iii) a C-terminal G3 domain comprising epidermal growth factor- (EGF-) like domains, a lectin-like domain, and a complement regulatory protein- (CRP-) like domain. The G2 domain, specific only for ACAN structure, comprises two PTR domains.

The hyaluronan binding domain, common in all four lecticans [36], is especially important during inflammatory process and tumor metastasis [37], while the CLDs are currently involved in processes like cell-cell adhesion [38] and immune response to pathogens [39-41].

Studies [30-32, 36, 41-44] focused on the functional features of NCAN showed that it binds to various ECM components, such as hyaluronan, heparin, tenascin- $\mathrm{C}$, and tenascin- $\mathrm{R}$, it is able to modulate the cell binding and neurite outgrowth, and it can also interact with neural cell adhesion molecule NCAM.

The interaction between the CLD of NCAN and tenascin$\mathrm{C}$ active domain was investigated by Rauch et al. [30] where the authors concluded the following: (i) NCAN CLD interacts with the pair of tenascin-C FnIII domains, namely, TNfn4 and TNfn5, in a divalent cation-dependent manner and (ii) NCAN-tenascin-C complex is able to modify the functional properties of tenascin-C.

Another important partner of NCAN is NCAM [31, 32], the first described member of cell adhesion receptors from the immunoglobulin superfamily. NCAM extracellular domain comprises five immunoglobulin (Ig) modules and three fibronectin type III modules (see Figure 2). Rao et al. [45] and Kasper et al. [46] showed that the Ig modules of
NCAM are critical for many features of NCAM like the hemophilic interaction of NCAM, affinity for the chondroitin sulfate binding site [47], and the interaction with oligosaccharides [48]. In order to elucidate the NCAN-NCAM mechanism of interaction, Rauch et al. [31] performed a very interesting study whose results showed that two NCAM residues located in the CLD, namely, methionine 773 and leucine 639, are critical for NCAM/NCAN interaction. These amino acids were able to increase NCAM capacity to interact with NCAN, while threonine in 950 reduced the capacity of NCAM to interact with NCAN. The study revealed the following: (i) NCAM interacts with all major NCAN domains in almost similar manner; (ii) NCAN active domains are not able to inhibit NCAM if they are dissociated from each other.

Regarding the brain expression of NCAN during embryonic and postnatal development, it was shown $[29,53,54]$ that, in rats, NCAN was first detected at the tenth embryonic day and that it had a maximum expression level around birth and decreased levels in the mature brain [29]. Zhou et al. [29] also showed that NCAN is expressed in several areas like thalamus, spinal cord, hypothalamus, or the cerebellum. Additional information was brought by Rauch et al. [30] which showed that NCAN interacts with tenascin-C in cerebellum at postnatal day 7 .

ACAN, another member of the lectican family of CSPGs, is an important constituent of perineuronal nets $[55,56]$. It is also distributed in the entire body, mostly in cartilage and brain, as aggregates formed with hyaluronic acid that binds to ACAN N-terminal domain [30]. It plays a major role in growth and homeostasis [57]. The domain structure of ACAN includes an $\mathrm{N}$-terminal domain, namely, G1, separated from a second globular domain (G2) by a short interglobular domain, an elongated domain carrying keratan sulfate and chondroitin sulfate chains, and a C-terminal globular G3 domain $[58,59]$ (see Figure 1). Specific for G1 domain is an Nterminal immunoglobulin-like repeat and two proteoglycan tandem repeats domains. These proteoglycan tandem repeats domains are responsible for the interaction of ACAN with hyaluronan [60]. It is also shown that G2 domain, found only in ACAN structure, contains two proteoglycan tandem repeats domains but these have no involvement in the interaction with hyaluronan [60]. The G3 domain comprises four structural motifs: two EGF-like repeats, CLD, and a complement regulatory protein (CRP or sushi) repeat [61]. The functions of EGF and CRP motifs of G3 are unknown, while CLD is involved in binding the carbohydrate [62] and in establishing calcium-dependent high affinity interactions with ECM proteins: tenascin- $\mathrm{R}$, tenascin-C, fibulin-1, fibulin2 , and fibrillin-1 [63]. ACAN genes identified as putative homologues belong to the following species: H. sapiens, $M$. mulatta, C. lupus, M. musculus, and R. norvegicus [33].

VCAN is another member of lectican family. Four isoforms of VCAN (V0, V1, V2, and V3) have been identified in various tissues, including the brain [64]. Structurally, all VCAN isoforms include an $\mathrm{N}$-terminal domain (G1), a GAG attachment region, and a C-terminal domain (G3) (see Figure 1). The exception is represented by V3 isoform, which has no GAG attachment region [64] but maintains its ability to bind hyaluronan through the G1 domain and to interact 


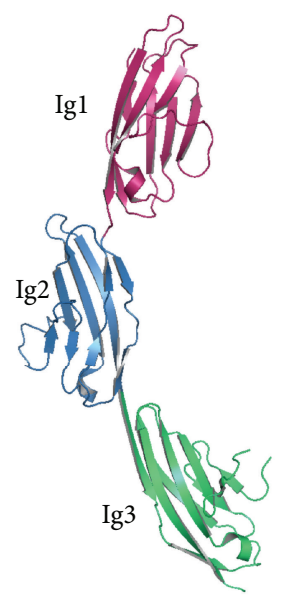

(a)

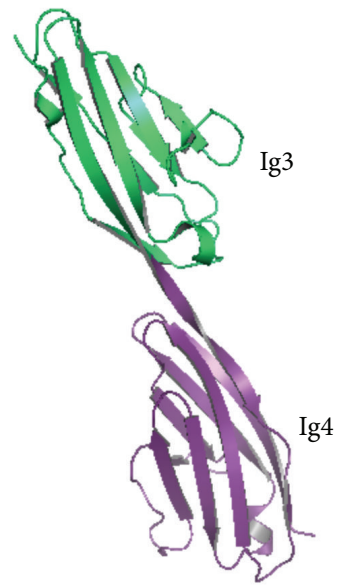

(b)

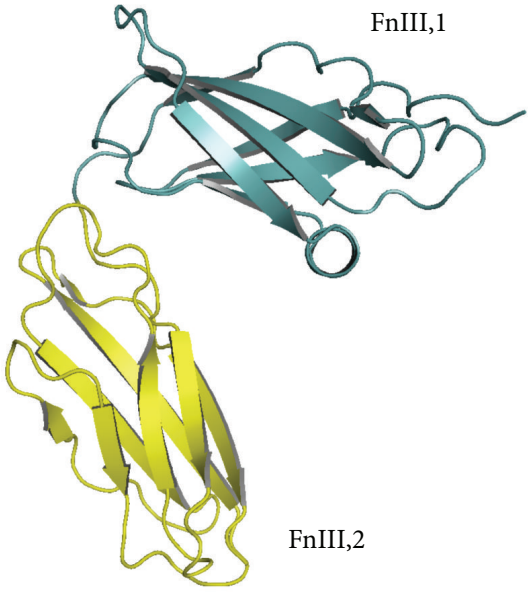

(c)

FIGURE 2: The crystal structures of the following NCAM fragments: (a) the first three N-terminal Ig modules (Ig1, Ig2, and Ig3), according to the crystal structure 1QZ1 [49]; (b) the third and fourth Ig modules (Ig3 and Ig4), according to the crystal structure 2XY1 [50]; and (c) first two fibronectin type III modules (FnIII,1 and FnIII,2), according to the crystal structure 2VKW [51]. The domains are labeled in the figure and are represented using different colors. Figures were made by Mernea M. using the free license software Pymol [52].

with EGF receptors through the EGF-like subdomains of G3 [65]. Similarly to NCAN, VCAN interacts with other ECM components through its three structural domains. Based on the interactions mediated by G1 and G3 domains, VCAN is able to regulate cell invasion and metastasis [66].

The studies presented in this section clearly suggest that, in the body, CSPGs expressions impose their functions and any mutation which appear at nucleotide sequence of CSPGs may induce changes in their functions. In the next section we will present changes in NCAN structure able to induce brain disorders.

\section{CSPGs Structure-Function Relationship in Genetic Disorders}

The expression of NCAN, VCAN, and ACAN in cells was associated with inhibition of axonal regeneration and neurogenesis after central nervous system injury $[8,9,16,20,67-$ 69]. Also, the presence of NCAN mutants was associated with psychiatric disorders as bipolar disorder, schizophrenia, and ADHD [17-19, 70-74].

Studies $[8,9,16,20,67-69]$ on the levels of NCAN, BCAN, and VCAN after spinal cord injury identified large upregulation of these CSPGs which leads to inhibition of axon growth. The upregulation is obvious as early as one day after injury and the return to normal expression levels occurs in 4 weeks after injury [8].

Many recent studies were focused on genome-wide significant association (GWSA) between variation in the NCAN gene expression and psychiatric disorders such as BD, schizophrenia, and ADHD [17-19, 70-74]. Recently, Mühleisen et al. [17], Cichon et al. [18], Schultz et al. [70], and Oruc et al. [19] performed GWSA studies between common variation in the NCAN gene (NCAN, rs1064395) and the psychiatric disorders BD and schizophrenia.
Mühleisen et al. [17] focused their research on identifying if $\mathrm{BD}$ and schizophrenia partly present common genetic risk factors in a large number of subjects (5061 patients and 9655 controls) genotyped for NCAN rs1064395 single-nucleotide polymorphism (SNP). Their results showed that rs1064395 A-allele, known to be a risk factor for BD, was significantly overrepresented in schizophrenia patients compared to control group [17]. These results, sustained by similar data $[18,19,70,75]$, suggest that genetic variation in NCAN rs1064395 represents a common risk factor for BD and schizophrenia. The patients with schizophrenia showed significantly increased rates of $\mathrm{BD}$ in comparison with control subjects. Furthermore, the study of schizophrenia elaborated by Mühleisen et al. was performed on independent samples, different from those used by the Psychiatric GWSA Consortium study [76], and showed that BD and schizophrenia share common etiological factors [17].

Schultz et al. [70] recently described the association between NCAN alleles and cortical thickness and folding in a number of 63 schizophrenia patients and 65 controls enrolled in their study. These subjects were genotyped for NCAN (rs1064395) SNP and were diagnosed using magnetic resonance imaging. The study results presented the following: (i) in patients with schizophrenia, NCAN risk represented by AA and AG carriers was found to be associated with higher folding in right lateral occipital and lesser in left dorsolateral prefrontal cortical areas; (ii) in control subjects, no such associations were detected; (iii) no significant association was determined in the case of cortical thickness of either patients or controls. The study results reconfirmed the involvement of NCAN in the visual processing and top-down cognitive activity, with these processes being severely disturbed in patients with schizophrenia.

Another significant GWSA study between NCAN gene markers and $\mathrm{BD}$ was performed by Cichon et al. [18]. 
In this study, authors investigated 499,494 autosomal and 12,484 X-chromosomal SNPs in a huge number of subjects (682 patients with BD and 1300 control subjects) from six different European countries. Interesting results were obtained when the correlation between $\mathrm{BD}$ and overexpression of NCAN genes was considered, showing that NCAN marker (rs1064395) is present in patients with BD. The relevant conclusions and perspective of the study were as follows: (i) NCAN is a potential BD susceptibility factor; (ii) localization of NCAN at the level of central nervous system (cortex, hippocampus), NCAN alleles, and BD susceptibility are positively correlated; and (iii) NCAN-deficient mice should be furthermore studied in order to identify the more subtle changes in the cognitive processes that are severely affected in $\mathrm{BD}$, like learning and memories. This idea is based on the observation [29] that maintenance of late-phase longterm potentiation in the hippocampal CA1 region in null mutants is reduced, which leads to mild deficits in learning and memory.

NCAN polymorphism association with BD was also investigated by Oruc et al. [19]. The aim of this study was to determine the relationship between NCAN polymorphism and genetic risk in a population sample from Bosnia and Herzegovina [19]. An important number of subjects represented by 56 patients and 30 healthy volunteers were genotyped for NCAN marker (rs1064395) using direct sequencing method. All three expected genotypes, GG, AG, and AA, were observed in this study. The obtained results were as follows: (i) allele AA appeared in frequencies of 1 in case group in 1 case and 0 in control group, and (ii) allele AG appeared in frequencies of 11 in case group in 5 cases and 0 in control group, while (iii) allele GG appeared in frequencies of 44 in case group in 21 cases and 0 in control group. Based on these results the authors concluded the following: (i) no genetic association was found between risk allele A for NCAN and schizophrenia; (ii) suggestive overexpression of risk alleles was found, but no statistical significance could be determined with standard statistical methods for genetic association analysis.

Very recently, Schimmelmann et al. [72] identified for the first time the risk alleles in children with ADHD and BD. Taking into account the high comorbidity of BD and ADHD, their phenotypic overlap especially in pediatric populations, the high heritability of both disorders, and the cooccurrence in families, the authors studied the possibility of the two disorders to share common genetic risk factors. The GWSA study was performed in 495 ADHD children and 1300 control subjects. No significant association was found between childhood ADHD and single BD risk alleles, but the polygene analysis for BD risk alleles at loci in NCAN, BCAN, and lectin, mannose binding 2-like gene, indicated a higher probability of a BD risk allele carrier to be associated with ADHD.

GWSA studies reviewed here presented the critical importance of NCAN marker (rs1064395 SNP) in identification of $\mathrm{BD}$ genetic risk and its possible role in $\mathrm{BD}$ association with other psychiatric disorders as schizophrenia and ADHD. Unfortunately, the mechanism by which NCAN (rs1064395) SNP could be involved in these psychiatric disorders is still unclear. Beside the clinical studies, in silico methods such as molecular modeling and computational mutagenesis techniques could establish a relationship between chemical structure and function of NCAN. These should bring supplementary knowledge on the mechanism by which single or multiple mutations in NCAN gene are able to induce BD genetic risk.

Even if at this time there are no in silico studies on the role of rs1064395 SNP in BD risk, based on our expertise in computational mutagenesis and quantitative structureactivity relationship (QSAR) applied to peptides and proteins $[79,80]$, we may suggest that the critical molecular features for the biological activity of NCAN native and its mutants should be represented by van der Waals surface and/or solvent accessible surface areas $[81,82]$ or count of atoms and bound types (e.g., polar and hydrophobic atoms, rigid and rotatable bonds) [83]. The substitution of a NCAN amino acid in a crucial position due to expression of a SNP may be able to change its molecular features, even if in a discreet manner, but sufficiently to induce changes in NCAN functionality during neuronal development.

Beside the elucidation of the mechanism by which CSPGs are involved in psychiatric disorders, another very important aspect of both clinical and in silico studies is represented by identification of mechanism by which the ligands are able to reduce CSPGs activity in the nervous system. Accordingly, clinical [76] and in silico studies [79, 80] focused on drugs involved in psychiatric disorders treatment as well as in reducing CSPGs (NCAN, ACAN, and VCAN) activity are presented in the next section. Also in the next section we present a brief description of in silico methods applied to CSPGs.

\section{CSPGs Structure-Function Relationship In Silico Approaches}

In silico research in medicine is thought to have the potential to speed the rate of discovery and reduce the need for expensive lab work and clinical trials. QSAR is used to obtain a strong relationship between the experimental biological activity of chemical structures and their structural features. QSAR is of great importancein the design of de novo drugs that present improvedpharmacokinetic and pharmacodynamic features with reduced side effects [79-81].

Recently, many research groups have applied QSAR techniques to develop new, more potent psychiatric drugs. An increasingly attractive strategy in pharmaceutical science is to discover new applications for drugs that are already clinically approved. For this aim the QSAR methods is an appropriate method.

In line with this aim, here we present QSAR studies applied to drugs used in schizophrenia treatment, namely, antipsychotics, with dual activities: inhibitory effect on lectican family [84] and also on membrane receptors [77, 85]. Schizophrenia symptoms are generally classified in two classes: positive (e.g., delusions, hallucinations, and disorganization) and negative symptoms (apathy, attention impairment, affective disorder, and several degrees of social 


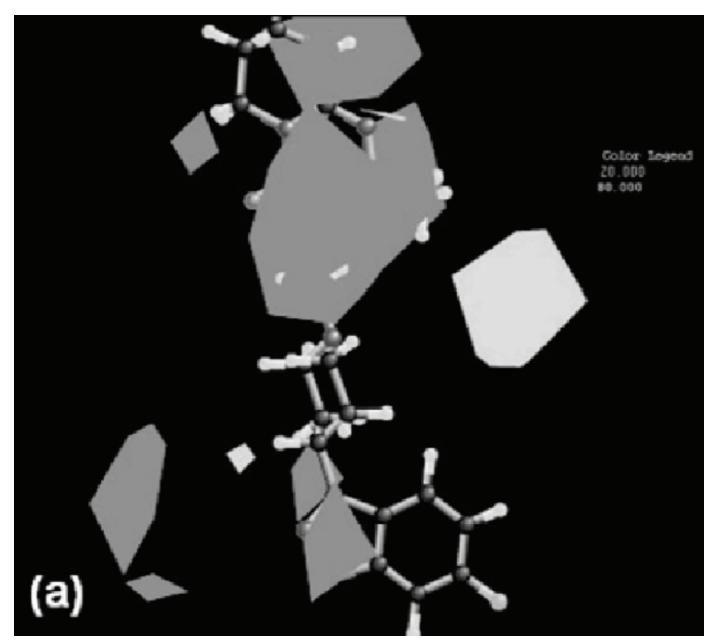

(a)

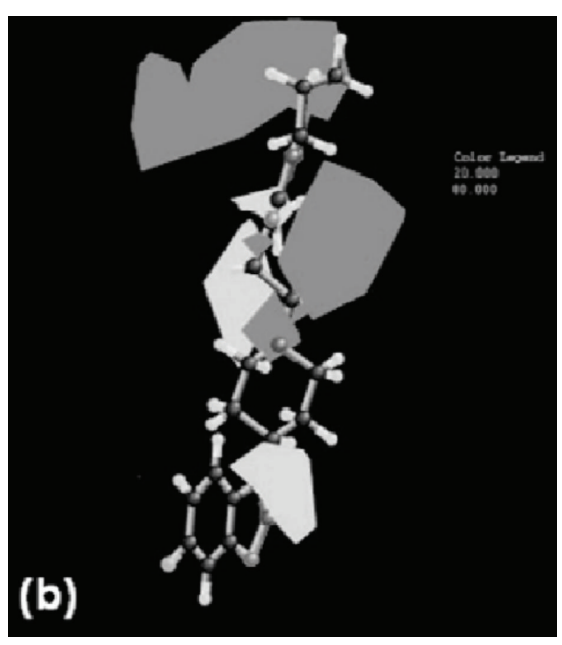

(b)

FIGURE 3: (a) Representation of the favorable (white polygons) and unfavorable (grey polygons) hydrophobic areas of risperidone (biological activity on D2 $=8.18$ ) when its antagonist potency at dopamine D2 receptor is considered and (b) representation of the favorable (white polyhedral) and unfavorable (grey polyhedral) hydrophobic areas of risperidone (biological activity on 5HT2A $=9.76$ ) when its antagonist potency at the serotonin 5HT2A receptor is considered [after Avram et al. [77], copyright permission].

impairment) [86]. Severe symptoms comprise psychosis, apathy and social withdrawal, important disturbance of professional skills, less independent life, or bad interpersonal relationships. Recent reports mention an increased number of patients diagnosed with psychoses with depression spectrum [87].

Avram et al. [77, 85, 88] applied advanced 3D QSAR methods to establish the most important descriptors that are critical for antipsychotic drugs interactions with membrane receptors like dopamine D1-D4, serotonin (5HT1A, 5HT2A), or adrenergic. One of these studies [77] has drawn several conclusions: (i) the hydrogen acceptor bond and the hydrophobic, electrostatic, and steric properties should be considered simultaneously to define the antagonist potency at the dopamine D2 receptor; (ii) the hydrogen bond donor and the hydrophobic, electrostatic, and steric properties should be considered simultaneously to define the antipsychotics affinity at the serotonin 5HT2A receptor; (iii) the design of new chemical structures with antagonistic activity on D2 and 5HT2A receptors could be improved by modulating their physicochemical properties, especially their hydrogen bond acceptor/donor and hydrophobic properties (Figure 3). For instance, risperidone derivatives with increased antagonist potency to the dopamine D2 receptor were obtained by increasing hydrophobic contacts on risperidone rings, while simultaneously retaining a fluorine atom [77].

Johnstone et al. [84] studied the antipsychotic drugs from phenothiazines (PhAPs) class such as prochlorperazine, fluphenazine, and trifluoperazine and non-PhAPs that may induce changes in cellular signalling and promote neurite outgrowth on inhibitory substrates. Some of these drugs are able to promote neurite outgrowth in cultured neurons in the presence of inhibitory CSPG substrate. The results revealed very important information: (i) trifluoperazine, prochlorperazine, perphenazine, and fluphenazine significantly enhanced neurite growth, branching, and axon length in hippocampal neurons on the CSPG substrates NCAN, VCAN, and ACAN; (ii) unexpected similar activity of piperazine $\mathrm{PhAPs}$ and a novel regeneration-promoting compound named F050 was noticed in increasing central nervous system neuronal outgrowth when growth was restricted by CSPGs; (iii) taking into account the severe side effects induced by $\mathrm{PhAP}$ antipsychotics, to use these structures for treatment of central nervous system injury imposes concerning dosage. In this study, for tested PhAP, there was a narrow concentration window between efficacy (growth promotion) and toxicity (cell death). Therefore, the authors suggested that the efforts could include future QSAR studies to identify compounds that promote growth without killing cells.

Other studies $[89,90]$ were focused on antipsychotics such as olanzapine, quetiapine, and clozapine that were able to enhance growth factor which induced neurite outgrowth in cell line PC12 from adrenal medulla, at concentrations of $10-40 \mu \mathrm{M}$ [89]. In the same study [89] clozapine and fluphenazine were shown to be able to increase axon lengths from mechanosensory neurons in Caenorhabditis elegans, at a concentration of $160 \mu \mathrm{M}$ [90].

These studies are also relevant to the mechanisms through which antipsychotics reduce the symptoms of psychosis since it was shown that schizophrenia is characterized by deficits in neuronal growth and connectivity [91], and genes associated with schizophrenia, such as disrupted in schizophrenia 1 (DISC1), are known to play significant roles in neurite growth. For example, Pantazopoulos et al. [92] showed that the levels of CSPGs are increased in postmortem schizophrenic brains. Therefore, the ability of antipsychotics to promote growth in the presence of glial-derived inhibitory molecules may represent a mechanism for improving neuronal connectivity in schizophrenic patients. 


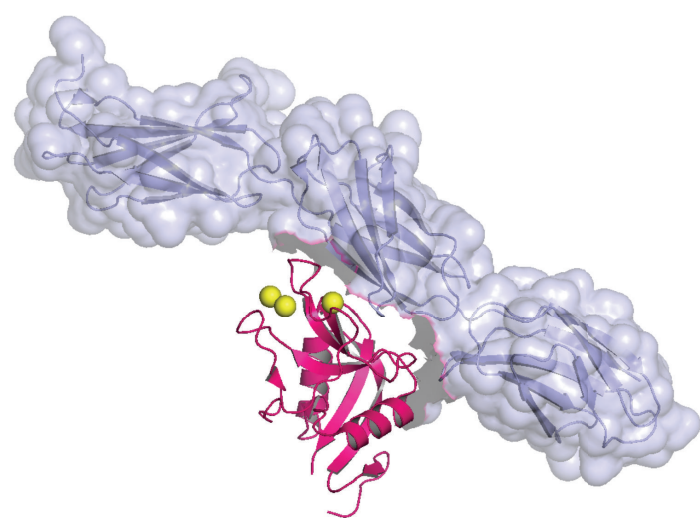

(a)

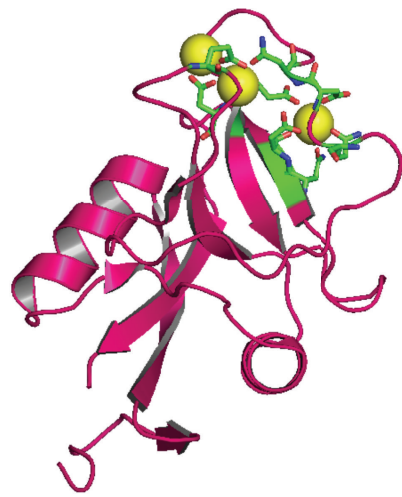

(b)

FIgURE 4: (a) The structure of ACAN core protein in complex with tenascin-R FnIII repeats $3-5$, as revealed by the crystal structure 1TDQ [78]. The tenascin- $\mathrm{R}$ fragment is represented as a light blue transparent surface that covers its backbone represented with the same color. ACAN core protein is represented with magenta and the three $\mathrm{Ca}^{2+}$ ions coordinated by ACAN are represented with yellow. (b) Detail on ACAN core protein (represented with magenta). The $\mathrm{Ca}^{2+}$ ions are represented with yellow and the residues involved in coordinating them are represented with green. Figures were made by Mernea M. using the free license software Pymol [52].

Other useful studies for identification of CSPGs structural changes are X-ray crystallography studies supplied by molecular modeling. Such a study was focused on ACAN [56, 78]. The structure of rat ACAN CLD domain was solved by $\mathrm{X}$-ray crystallography $\left(2.6 \AA\right.$ resolution) in a $\mathrm{Ca}^{2+}$-dependent complex with fibronectin type III (FnIII) repeats $3-5$ of rat tenascin-R [78] (see Figure 4(a)). Tenascin-R and CSPGs present a colocalized expression in the nervous system and form specialized ECM structures called perineuronal nets [93]. Multimeric tenascins act as molecular cross-linkers between CSPG-hyaluronan complexes and the evidence of network formation in vivo was obtained by Lundell et al. [78] using electron microscopy. Molecular evidence of this interaction is brought by the crystal structure deposited in Protein Data Bank under the code 1TDQ [78] solved by the same authors. In this structure, ACAN core protein comprises 126 residues and has three disulfide bridges: Cys3Cys14, Cys31-Cys123, and Cys99-Cys115. The last disulphide bridge is located at $8 \AA$ distance from two $\mathrm{Ca}^{2+}$ ions. All residues are numbered according to the fragment in the crystal structure.

The structure 1TDQ comprises a total of three $\mathrm{Ca}^{2+}$ ions, with all of them being coordinated by ACAN residues (see Figure 4(b)). The carbohydrate binding $\mathrm{Ca}^{2+}$ ion, conserved in all structures of CLDs, is coordinated by amino acids: Gln88, Asp90, Glu97, Asn111, and Asp112 and by a water molecule. A second $\mathrm{Ca}^{2+}$ ion is coordinated by amino acids: Glu68, Asn91, Glu97, and Asp98. The third $\mathrm{Ca}^{2+}$ ion is coordinated by amino acids Asp98 and Glu68. The last two $\mathrm{Ca}^{2+}$ ions are not constant in solved CLDs structures. The $\mathrm{Ca}^{2+}$ ions are involved in ordering the neighboring ACAN loops and it was noticed [78] that these loops further make specific interactions with one of the FnIII repeats of tenascin$\mathrm{R}$.

In complex with the FnIII domain of rat tenascin-R, the $\beta 6$ and $\beta 7$ strands of ACAN and also its extended loop
L4 are buried [78]. The contact area between ACAN and tenascin- $\mathrm{R}$ is free of carbohydrate. The interaction surface of ACAN is composed of 55\% polar and $45 \%$ nonpolar residues and can be divided into three regions. Near $\mathrm{Ca}^{2+}$ ions, the surface is mostly hydrophobic, with residues Phe92 and Phe93 from L4 loop being of particular interest for the interaction with tenascin. In other CLD structures such as that of mannose binding protein, the homologous residue of Phe92 is an aromatic residue which interacts with the bound carbohydrate [90]. Other residues important for the interaction with tenascin are (i) Val101, Tyrl17, and Leul19, which form the hydrophobic pocket that accommodates residue Leul31 from tenascin structure; (ii) residue Asn111 which forms a hydrogen bond with tenascin Leul31; (iii) residue Arg21 which forms two salt bridges with tenascin Glu161; (iv) Asp56 that makes a salt bridge with tenascin Arg183; (v) Asn53 and Asn54 forming hydrogen bonds that block residue Arg260 from tenascin structure; (vi) Trp104 which interacts with a tenascin hydrophobic pocket; and (vii) Gln118 which forms a hydrogen with tenascin Asn181 [78].

\section{Conclusion and Perspective}

We presented here a number of experimental and in silico studies focused on characterizing the structural and functional features of CSPGs and their possible inhibitors. All reviewed studies considered the important role of CSPGs (e.g., NCAN, ACAN, VCAN, and BCAN) in physiological cellular processes and also in promoting disorders such as those associated with inhibition of axonal regeneration and neurogenesis after central nervous system injury and psychiatric disorders such as BD, schizophrenia, and ADHD.

Being localized in the neural ECM, these CSPGs have an important role in neuronal growth mechanisms represented by neuroplasticity, axon guidance, or neuron repair processes following injury to the spinal cord or brain. The discussed 
studies have a major goal to underline the correlation between the structural sequences and/or overexpression of these CSPG and brain disorders. Even if the number of preclinical and clinical studies is significant in this field, the mechanism by which NCAN, VCAN, or ACAN affects human tissues and brain is not enough understood.

For a better explanation of CSPG involvement in cellular processes, recent studies used structural and crystallographic methods as well as QSAR complementary techniques over the experimental methods such as GWSA. These studies were focused on the identification of structural changes underlining the function modification induced by changes in NCAN, VCAN, or ACAN sequences.

In perspective, the simultaneous use of experimental and in silico (computational mutagenesis, QSAR on proteins) techniques will allow more reliable early identification of CSPGs genes expression errors and will open the possibility to develop new population screening tests for susceptibility to the development of new and misdiagnosed neurodevelopment disorders.

What is more, by using computational mutagenesis, rational protein design, and the prediction of structuresproteins function relationship, it will be possible to study the structure of NCAN, VCAN, or ACAN mutants which are able or not to induce neurodevelopment disorders.

Considering the already established role of NCAN SNP (rs1064395) as risk marker for BD and schizophrenia disorders, by using QSAR methods we will be able to present the critical molecular features of NCAN SNP (rs1064395) in psychiatric disorders. Besides, QSAR could also be helpful in identifying molecular features of other possible NCAN SNPs markers for psychiatric disorders. On the other hand, these descriptors can be compared with the identical molecular features of native NCAN in order to notice the discreet changes in the structure and functionally of NCAN. We are confident that QSAR is an appropriate method to determine discreet changes in the molecular features of NCAN or other CSPGs.

\author{
Abbreviation \\ ACAN: Aggrecan \\ ADHD: Attention deficit hyperactivity disorder \\ BCAN: Brevican \\ BD: Bipolar disorder \\ CLD: C-type lectin domain \\ CRP: Complement regulatory protein \\ CSPGs: Chondroitin sulfate proteoglycans \\ ECM: Extracellular matrix molecules \\ EGF: Epidermal growth factor \\ GAG: Glycosaminoglycan \\ GWSA: Genome-wide significant association \\ NCAM: Neuronal cell adhesion molecule \\ NCAN: Neurocan \\ QSAR: Quantitative structure-activity relationship \\ PhAPs: Phenothiazines \\ SNP: $\quad$ Single-nucleotide polymorphism \\ VCAN: Versican.
}

\section{Conflict of Interests}

The authors declare that there is no conflict of interests regarding the publication of this paper.

\section{Acknowledgments}

Speranta Avram acknowledges the financial support of Romanian National Authority for Scientific Research through the UEFISCDI PNII Grants PCCA-89/2012 and PCCA16/2012. Maria Mernea acknowledges the financial support of Romanian National Authority for Scientific Research through the UEFISCDI PNII Grants PCCA-89/2012 and PCE-137/2011.

\section{References}

[1] M. Geissler, C. Gottschling, A. Aguado et al., "Primary hippocampal neurons, which lack four crucial extracellular matrix molecules, display abnormalities of synaptic structure and function and severe deficits in perineuronal net formation," The Journal of Neuroscience, vol. 33, no. 18, pp. 7742-7755, 2013.

[2] G. Perea, M. Navarrete, and A. Araque, "Tripartite synapses: astrocytes process and control synaptic information," Trends in Neurosciences, vol. 32, no. 8, pp. 421-431, 2009.

[3] M. A. Di Castro, J. Chuquet, N. Liaudet et al., "Local $\mathrm{Ca}^{2+}$ detection and modulation of synaptic release by astrocytes," Nature Neuroscience, vol. 14, no. 10, pp. 1276-1284, 2011.

[4] Y. Bekku and T. Oohashi, "Neurocan contributes to the molecular heterogeneity of the perinodal ECM," Archives of Histology and Cytology, vol. 73, no. 2, pp. 95-102, 2011.

[5] S. Wiese, M. Karus, and A. Faissner, "Astrocytes as a source for extracellular matrix molecules and cytokines," Frontiers in Pharmacology, vol. 3, p. 120, 2012.

[6] C. E. Bandtlow and D. R. Zimmermann, "Proteoglycans in the developing brain: new conceptual insights for old proteins," Physiological Reviews, vol. 80, no. 4, pp. 1267-1290, 2000.

[7] J. H. Yi, Y. Katagiri, B. Susarla, D. Figge, A. J. Symes, and H. M. Geller, "Alterations in sulfated chondroitin glycosaminoglycans following controlled cortical impact injury in mice," Journal of Comparative Neurology, vol. 520, no. 15, pp. 3295-3313, 2012.

[8] L. L. Jones, R. U. Margolis, and M. H. Tuszynski, "The chondroitin sulfate proteoglycans neurocan, brevican, phosphacan, and versican are differentially regulated following spinal cord injury," Experimental Neurology, vol. 182, no. 2, pp. 399-411, 2003.

[9] P. P. Monnier, A. Sierra, J. M. Schwab, S. Henke-Fahle, and B. K. Mueller, "The Rho/ROCK pathway mediates neurite growth-inhibitory activity associated with the chondroitin sulfate proteoglycans of the CNS glial scar," Molecular and Cellular Neuroscience, vol. 22, no. 3, pp. 319-330, 2003.

[10] B. Meyer-Puttlitz, P. Milev, E. Junker, I. Zimmer, R. U. Margolis, and R. K. Margolis, "Chondroitin sulfate and chondroitin/keratan sulfate proteoglycans of nervous tissue: developmental changes of neurocan and phosphacan," Journal of Neurochemistry, vol. 65, no. 5, pp. 2327-2337, 1995.

[11] S. Dutt, E. Cassoly, M. T. Dours-Zimmermann, M. Matasci, E. T. Stoeckli, and D. R. Zimmermann, "Versican V0 and V1 direct the growth of peripheral axons in the developing chick hindlimb," Journal of Neuroscience, vol. 31, no. 14, pp. 5262-5270, 2011. 
[12] T. L. Laabs, H. Wang, Y. Katagiri, T. McCann, J. W. Fawcett, and H. M. Geller, "Inhibiting glycosaminoglycan chain polymerization decreases the inhibitory activity of astrocyte-derived chondroitin sulfate proteoglycans," Journal of Neuroscience, vol. 27, no. 52, pp. 14494-14501, 2007.

[13] N. Bukhari, L. Torres, J. K. Robinson, and S. E. Tsirka, "Axonal regrowth after spinal cord injury via chondroitinase and the tissue plasminogen activator (tPA)/plasmin system," Journal of Neuroscience, vol. 31, no. 42, pp. 14931-14943, 2011.

[14] D. Carulli, T. Pizzorusso, J. C. F. Kwok et al., "Animals lacking link protein have attenuated perineuronal nets and persistent plasticity," Brain, vol. 133, no. 8, pp. 2331-2347, 2010.

[15] D. Wang, R. M. Ichiyama, R. Zhao, M. R. Andrews, and J. W. Fawcett, "Chondroitinase combined with rehabilitation promotes recovery of forelimb function in rats with chronic spinal cord injury," Journal of Neuroscience, vol. 31, no. 25, pp. 9332-9344, 2011.

[16] H. J. Lee, S. Bian, I. Jakovcevski, B. Wu, A. Irintchev, and M. Schachner, "Delayed applications of L1 and chondroitinase $\mathrm{ABC}$ promote recovery after spinal cord injury," Journal of Neurotrauma, vol. 29, no. 10, pp. 1850-1863, 2012.

[17] T. W. Mühleisen, M. Mattheisen, J. Strohmaier et al., "Association between schizophrenia and common variation in neurocan (NCAN), a genetic risk factor for bipolar disorder," Schizophrenia Research, vol. 138, no. 1, pp. 69-73, 2012.

[18] S. Cichon, T. W. Mühleisen, F. A. Degenhardt et al., "Genomewide association study identifies genetic variation in neurocan as a susceptibility factor for bipolar disorder," American Journal of Human Genetics, vol. 88, no. 3, pp. 372-381, 2011.

[19] L. Oruc, L. Kapur-Pojskic, J. Ramic, N. Pojskic, and K. Bajrovic, "Assessment of relatedness between neurocan gene as bipolar disorder susceptibility locus and schizophrenia," Bosnian Journal of Basic Medical Sciences, vol. 12, no. 4, pp. 245-248, 2012.

[20] K. Iseki, S. Hagino, T. Nikaido et al., "Gliosis-specific transcription factor OASIS coincides with proteoglycan core protein genes in the glial scar and inhibits neurite outgrowth," BioMed Research, vol. 33, no. 6, pp. 345-353, 2012.

[21] H. Kitagawa, T. Uyama, and K. Sugahara, "Molecular cloning and expression of a human chondroitin synthase," The Journal of Biological Chemistry, vol. 276, no. 42, pp. 38721-38726, 2001.

[22] H. Kitagawa, T. Izumikawa, T. Uyama, and K. Sugahara, "Molecular cloning of a chondroitin polymerizing factor that cooperates with chondroitin synthase for chondroitin polymerization," The Journal of Biological Chemistry, vol. 278, no. 26, pp. 23666-23671, 2003.

[23] T. Izumikawa, T. Koike, S. Shiozawa, K. Sugahara, J.-I. Tamura, and H. Kitagawa, "Identification of chondroitin sulfate glucuronyltransferase as chondroitin synthase-3 involved in chondroitin polymerization: chondroitin polymerization is achieved by multiple enzyme complexes consisting of chondroitin synthase family members," The Journal of Biological Chemistry, vol. 283, no. 17, pp. 11396-11406, 2008.

[24] T. Izumikawa, T. Uyama, Y. Okuura, K. Sugahara, and H. Kitagawa, "Involvement of chondroitin sulfate synthase-3 (chondroitin synthase-2) in chondroitin polymerization through its interaction with chondroitin synthase-1 or chondroitinpolymerizing factor," Biochemical Journal, vol. 403, no. 3, pp. 545-552, 2007.

[25] T. Mikami and H. Kitagawa, "Biosynthesis and function of chondroitin sulfate," Biochim Biophys Acta, 1830, no. 10, pp. 4719-4733, 2013.
[26] A. Hurtado, H. Podinin, M. Oudega, and B. Grimpe, "Deoxyribozyme-mediated knockdown of xylosyltransferase-1 mRNA promotes axon growth in the adult rat spinal cord," Brain, vol. 131, no. 10, pp. 2596-2605, 2008.

[27] R. C. Cua, L. W. Lau, M. B. Keough, R. Midha, S. S. Apte, and V. W. Yong, "Overcoming neurite-inhibitory chondroitin sulfate proteoglycans in the astrocyte matrix," Glia, vol. 61, no. 6, pp. 972-984, 2013.

[28] C. K. Prange, L. A. Pennacchio, K. Lieuallen, W. Fan, and G. G. Lennon, "Characterization of the human neurocan gene, CSPG3," Gene, vol. 221, no. 2, pp. 199-205, 1998.

[29] X.-H. Zhou, C. Brakebusch, H. Matthies et al., "Neurocan is dispensable for brain development," Molecular and Cellular Biology, vol. 21, no. 17, pp. 5970-5978, 2001.

[30] U. Rauch, A. Clement, C. Retzler et al., "Mapping of a defined neurocan binding site to distinct domains of tenascin-C," The Journal of Biological Chemistry, vol. 272, no. 43, pp. 2690526912, 1997.

[31] U. Rauch, K. Feng, and X.-H. Zhou, "Neurocan: a brain chondroitin sulfate proteoglycan," Cellular and Molecular Life Sciences, vol. 58, no. 12-13, pp. 1842-1856, 2001.

[32] C. Retzler, W. Gohring, and U. Rauch, "Analysis of neurocan structures interacting with the neural cell adhesion molecule NCAM," The Journal of Biological Chemistry, vol. 271, no. 44, pp. 27304-27310, 1996.

[33] L. Y. Geer, A. Marchler-Bauer, R. C. Geer et al., "The NCBI BioSystems database," Nucleic Acids Research, vol. 38, no. 1, pp. D492-D496, 2009.

[34] M. Magrane and U. Consortium, "UniProt Knowledgebase: a hub of integrated protein data," Database, vol. 2011, p. bar009, 2011.

[35] R. V. Iozzo and A. D. Murdoch, "Proteoglycans of the extracellular environment: clues from the gene and protein side offer novel perspectives in molecular diversity and function," The FASEB Journal, vol. 10, no. 5, pp. 598-614, 1996.

[36] N. T. Seyfried, G. F. McVey, A. Almond, D. J. Mahoney, J. Dudhia, and A. J. Day, "Expression and purification of functionally active hyaluronan-binding domains from human cartilage link protein, aggrecan and versican: formation of ternary complexes with defined hyaluronan oligosaccharides," The Journal of Biological Chemistry, vol. 280, no. 7, pp. 54355448, 2005.

[37] P. Teriete, S. Banerji, M. Noble et al., "Structure of the regulatory hyaluronan binding domain in the inflammatory leukocyte homing receptor CD44," Molecular Cell, vol. 13, no. 4, pp. 483496, 2004.

[38] E. M. Wood-Charlson and V. M. Weis, "The diversity of Ctype lectins in the genome of a basal metazoan, Nematostella vectensis," Developmental and Comparative Immunology, vol. 33, no. 8, pp. 881-889, 2009.

[39] Y. van Kooyk, "C-type lectins on dendritic cells: key modulators for the induction of immune responses," Biochemical Society Transactions, vol. 36, no. 6, pp. 1478-1481, 2008.

[40] M. Cardone, K. N. Ikeda, B. Varano et al., "Opposite regulatory effects of IFN-beta and IL-3 on C-type lectin receptors, antigen uptake, and phagocytosis in human macrophages," Journal of Leukocyte Biology, vol. 95, no. 1, pp. 161-168, 2014.

[41] K. Gijzen, A. Cambi, R. Torensma, and C. G. Figdor, "C-type lectins on dendritic cells and their interaction with pathogenderived and endogenous glycoconjugates," Current Protein and Peptide Science, vol. 7, no. 4, pp. 283-294, 2006. 
[42] M. Pyka, C. Wetzel, A. Aguado, M. Geissler, H. Hatt, and A. Faissner, "Chondroitin sulfate proteoglycans regulate astrocytedependent synaptogenesis and modulate synaptic activity in primary embryonic hippocampal neurons," European Journal of Neuroscience, vol. 33, no. 12, pp. 2187-2202, 2011.

[43] L. Gasimli, H. E. Stansfield, A. V. Nairn et al., "Structural remodeling of proteoglycans upon retinoic acid-induced differentiation of NCCIT cells," Glycoconjugate Journal, vol. 30, no. 5, pp. 497-510, 2013.

[44] S. Hoffman, B. C. Sorkin, P. C. White et al., "Chemical characterization of a neural cell adhesion molecule purified from embryonic brain membranes," The Journal of Biological Chemistry, vol. 257, no. 13, pp. 7720-7729, 1982.

[45] Y. Rao, X.-F. Wu, J. Gariepy, U. Rutishauser, and C.-H. Siu, "Identification of a peptide sequence involved in homophilic binding in the neural cell adhesion molecule NCAM," Journal of Cell Biology, vol. 118, no. 4, pp. 937-949, 1992.

[46] C. Kasper, H. Rasmussen, J. S. Kastrup et al., "Structural basis of cell-cell adhesion by NCAM," Nature Structural Biology, vol. 7, no. 5, pp. 389-393, 2000.

[47] O. Nybroe, N. Moran, and E. Bock, "Equilibrium binding analysis of neural cell adhesion molecule binding to heparin," Journal of Neurochemistry, vol. 52, no. 6, pp. 1947-1949, 1989.

[48] R. Horstkorte, M. Schachner, J. P. Magyar, T. Vorherr, and B. Schmitz, "The fourth immunoglobulin-like domain of NCAM contains a carbohydrate recognition domain for oligomannosidic glycans implicated in association with $\mathrm{L} 1$ and neurite outgrowth," Journal of Cell Biology, vol. 121, no. 6, pp. 1409-1422, 1993.

[49] V. Soroka, K. Kolkova, J. S. Kastrup et al., "Structure and interactions of NCAM Ig1-2-3 suggest a novel zipper mechanism for homophilic adhesion," Structure, vol. 11, no. 10, pp. 1291-1301, 2003.

[50] N. Kulahin, O. Kristensen, K. K. Rasmussen et al., "Structural model and trans-interaction of the entire ectodomain of the olfactory cell adhesion molecule," Structure, vol. 19, no. 2, pp. 203-211, 2011.

[51] F. Carafoli, J. L. Saffell, and E. Hohenester, "Structure of the tandem fibronectin type 3 domains of neural cell adhesion molecule," Journal of Molecular Biology, vol. 377, no. 2, pp. 524534, 2008.

[52] The PyMOL Molecular Graphics System, Version 1.5.0.4, Schrödinger, LLC..

[53] N. B. Schwartz and M. Domowicz, "Proteoglycans in brain development," Glycoconjugate Journal, vol. 21, no. 6, pp. 329341, 2004.

[54] S. Popp, P. Maurel, J. S. Andersen, and R. U. Margolis, “Developmental changes of aggrecan, versican and neurocan in the retina and optic nerve," Experimental Eye Research, vol. 79, no. 3, pp. 351-356, 2004.

[55] M. Morawski, G. Brückner, T. Arendt, and R. T. Matthews, "Aggrecan: beyond cartilage and into the brain," International Journal of Biochemistry and Cell Biology, vol. 44, no. 5, pp. 690693, 2012.

[56] A. Aspberg, "The different roles of aggrecan interaction domains," Journal of Histochemistry \& Cytochemistry, vol. 60, no. 12, pp. 987-996, 2012.

[57] A. D. Theocharis, S. S. Skandalis, G. N. Tzanakakis, and N. K. Karamanos, "Proteoglycans in health and disease: novel roles for proteoglycans in malignancy and their pharmacological targeting," FEBS Journal, vol. 277, no. 19, pp. 3904-3923, 2010.
[58] H. Wiedemann, M. Paulsson, and R. Timpl, "Domain structure of cartilage proteoglycans revealed by rotary shadowing of intact and fragmented molecules," Biochemical Journal, vol. 224, no. 1, pp. 331-333, 1984.

[59] M. Paulsson, M. Mörgelin, H. Wiedemann et al., "Extended and globular protein domains in cartilage proteoglycans," Biochemical Journal, vol. 245, no. 3, pp. 763-772, 1987.

[60] H. Watanabe, S. C. Cheung, N. Itano, K. Kimata, and Y. Yamada, "Identification of hyaluronan-binding domains of aggrecan," The Journal of Biological Chemistry, vol. 272, no. 44, pp. 2805728065, 1997.

[61] H. Yamada, K. Watanabe, M. Shimonaka, and Y. Yamaguchi, "Molecular cloning of brevican, a novel brain proteoglycan of the aggrecan/versican family," The Journal of Biological Chemistry, vol. 269, no. 13, pp. 10119-10126, 1994.

[62] S. Saleque, N. Ruiz, and K. Drickamer, "Expression and characterization of a carbohydrate-binding fragment of rat aggrecan," Glycobiology, vol. 3, no. 2, pp. 185-190, 1993.

[63] R. Miura, A. Aspberg, I. M. Ethell et al., "The proteoglycan lectin domain binds sulfated cell surface glycolipids and promotes cell adhesion," The Journal of Biological Chemistry, vol. 274, no. 16, pp. 11431-11438, 1999.

[64] A. D. Theocharis, "Versican in health and disease," Connective Tissue Research, vol. 49, no. 3-4, pp. 230-234, 2008.

[65] K. Matsumoto, M. Shionyu, M. Go et al., "Distinct interaction of versican/PG-M with hyaluronan and link protein," The Journal of Biological Chemistry, vol. 278, no. 42, pp. 41205-41212, 2003.

[66] A. J. M. Yee, M. Akens, B. L. Yang et al., "The effect of versican G3 domain on local breast cancer invasiveness and bony metastasis," Breast Cancer Research, vol. 9, no. 4, article R42, 2007.

[67] J. R. Siebert and D. J. Osterhout, "The inhibitory effects of chondroitin sulfate proteoglycans on oligodendrocytes," Journal of Neurochemistry, vol. 119, no. 1, pp. 176-188, 2011.

[68] C. Jager, D. Lendvai, G. Seeger et al., "Perineuronal and perisynaptic extracellular matrix in the human spinal cord," Neuroscience, vol. 238, pp. 168-184, 2013.

[69] R. Tauchi, S. Imagama, T. Natori et al., "The endogenous proteoglycan-degrading enzyme ADAMTS-4 promotes functional recovery after spinal cord injury," Journal of Neuroinflammation, vol. 9, article 53, 2012.

[70] C. C. Schultz, T. W. Mühleisen, I. Nenadic et al., "Common variation in NCAN, a risk factor for bipolar disorder and schizophrenia, influences local cortical folding in schizophrenia," Psychological Medicine, vol. 44, no. 4, pp. 811-820, 2014.

[71] X. Miro, S. Meier, M. L. Dreisow et al., "Studies in humans and mice implicate neurocan in the etiology of mania," American Journal of Psychiatry, vol. 169, no. 9, pp. 982-990, 2012.

[72] B. G. Schimmelmann, A. Hinney, A. Scherag et al., "Bipolar disorder risk alleles in children with ADHD," Journal of Neural Transmission, vol. 120, no. 11, pp. 1611-1617, 2013.

[73] N. Craddock and P. Sklar, "Genetics of bipolar disorder," The Lancet, vol. 381, no. 9878, pp. 1654-1662, 2013.

[74] K. W. Lee, P. S. Woon, Y. Y. Teo, and K. Sim, "Genome wide association studies (GWAS) and copy number variation (CNV) studies of the major psychoses: what have we learnt?" Neuroscience and Biobehavioral Reviews, vol. 36, no. 1, pp. 556$571,2012$.

[75] J. X. Van Snellenberg and T. De Candia, "Meta-analytic evidence for familial coaggregation of schizophrenia and bipolar disorder," Archives of General Psychiatry, vol. 66, no. 7, pp. 748-755, 2009. 
[76] "Genome-wide association study identifies five new schizophrenia loci," Nature Genetics, vol. 43, no. 10, pp. 969-976, 2011.

[77] S. Avram, D. Duda-Seiman, F. Borcan, and P. Wolschann, "QSAR-CoMSIA applied to antipsychotic drugs with their dopamine D2 and serotonine 5HT2A membrane receptors," Journal of the Serbian Chemical Society, vol. 76, no. 2, pp. 263281, 2011.

[78] A. Lundell, A. I. Olin, M. Mörgelin, S. Al-Karadaghi, A. Aspberg, and D. T. Logan, "Structural basis for interactions between tenascins and lectican C-type lectin domains: evidence for a crosslinking role for tenascins," Structure, vol. 12, no. 8, pp. 1495-1506, 2004.

[79] S. Avram, D. Duda-Seiman, F. Borcan, B. Radu, C. DudaSeiman, and D. Mihailescu, "Evaluation of antimicrobial activity of new mastoparan derivatives using QSAR and computational mutagenesis," International Journal of Peptide Research and Therapeutics, vol. 17, no. 1, pp. 7-17, 2011.

[80] O. Calborean, M. Mernea, S. Avram, and D. F. Mihailescu, "Pharmacological descriptors related to the binding of Gp120 to CD4 corresponding to 60 representative HIV-1 strains," Journal of Enzyme Inhibition and Medicinal Chemistry, vol. 28, no. 5, pp. 1015-1025, 2013.

[81] S. Bhat and E. O. Purisima, "Molecular surface generation using a variable-radius solvent probe," Proteins: Structure, Function and Genetics, vol. 62, no. 1, pp. 244-261, 2006.

[82] A. J. Li and R. Nussinov, "A set of van der Waals and coulombic radii of protein atoms for molecular and solvent-accessible surface calculation, packing evaluation, and docking," Proteins, vol. 32, no. 1, pp. 111-127, 1998.

[83] T. I. Oprea, "Property distribution of drug-related chemical databases," Journal of Computer-Aided Molecular Design, vol. 14, no. 3, pp. 251-264, 2000.

[84] A. L. Johnstone, G. W. Reierson, R. P. Smith, J. L. Goldberg, V. P. Lemmon, and J. L. Bixby, "A chemical genetic approach identifies piperazine antipsychotics as promoters of CNS neurite growth on inhibitory substrates," Molecular and Cellular Neuroscience, vol. 50, no. 2, pp. 125-135, 2012.

[85] S. Avram, H. Berner, A. L. Milac, and P. Wolschann, "Quantitative structure-activity relationship studies on membrane receptors inhibition by antipsychotic drugs. Application to schizophrenia treatment," Monatshefte für Chemie, vol. 139, no. 4, pp. 407-426, 2008.

[86] W. T. Carpenter Jr., “The deficit syndrome," American Journal of Psychiatry, vol. 151, no. 3, pp. 327-329, 1994.

[87] I. V. Vahia, N. M. Lanouette, S. Golshan et al., "Adding antidepressants to antipsychotics for treatment of subsyndromal depressive symptoms in schizophrenia: impact on positive and negative symptoms," Indian Journal of Psychiatry, vol. 55, no. 2, pp. 144-148, 2013.

[88] S. Avram, A.-L. Milac, and D. Mihailescu, "3D-QSAR study indicates an enhancing effect of membrane ions on psychiatric drugs targeting serotonin receptor 5-HT1A," Molecular BioSystems, vol. 8, no. 5, pp. 1418-1425, 2012.

[89] X.-H. Lu and D. S. Dwyer, "Second-generation antipsychotic drugs, olanzapine, quetiapine, and clozapine enhance neurite outgrowth in PC12 cells via PI3K/AKT, ERK, and pertussis toxin-sensitive pathways," Journal of Molecular Neuroscience, vol. 27, no. 1, pp. 43-64, 2005.

[90] D. R. Donohoe, K. Weeks, E. J. Aamodt, and D. S. Dwyer, "Antipsychotic drugs alter neuronal development including ALM neuroblast migration and PLM axonal outgrowth in
Caenorhabditis elegans," International Journal of Developmental Neuroscience, vol. 26, no. 3-4, pp. 371-380, 2008.

[91] A. Zalesky, A. Fornito, M. L. Seal et al., "Disrupted axonal fiber connectivity in schizophrenia," Biological Psychiatry, vol. 69, no. 1, pp. 80-89, 2011.

[92] H. Pantazopoulos, T.-U. W. Woo, M. P. Lim, N. Lange, and S. Berretta, "Extracellular matrix-glial abnormalities in the amygdala and entorhinal cortex of subjects diagnosed with schizophrenia," Archives of General Psychiatry, vol. 67, no. 2, pp. 155-166, 2010.

[93] Y. Yamaguchi, "Lecticans: organizers of the brain extracellular matrix," Cellular and Molecular Life Sciences, vol. 57, no. 2, pp. 276-289, 2000. 

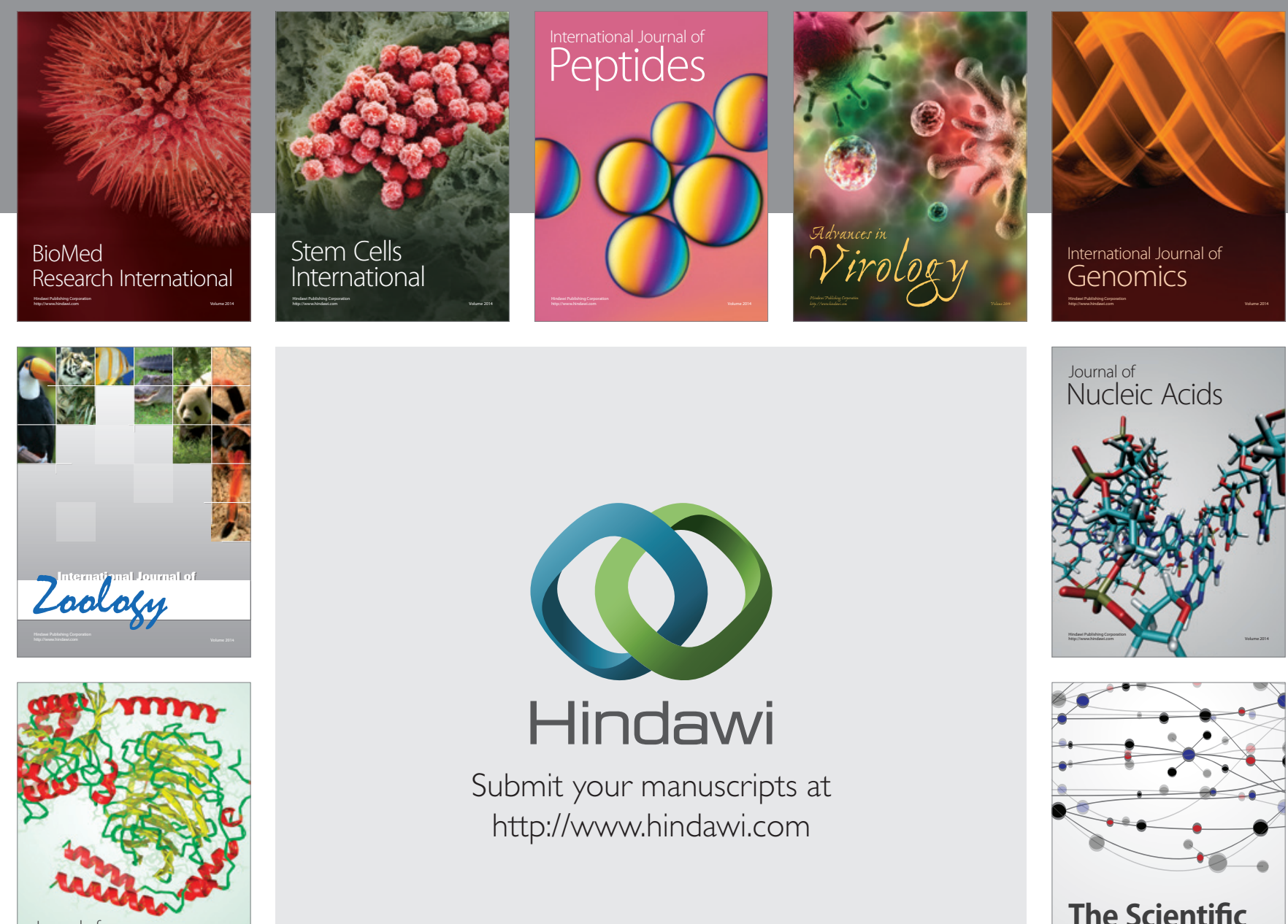

Submit your manuscripts at

http://www.hindawi.com

Journal of
Signal Transduction
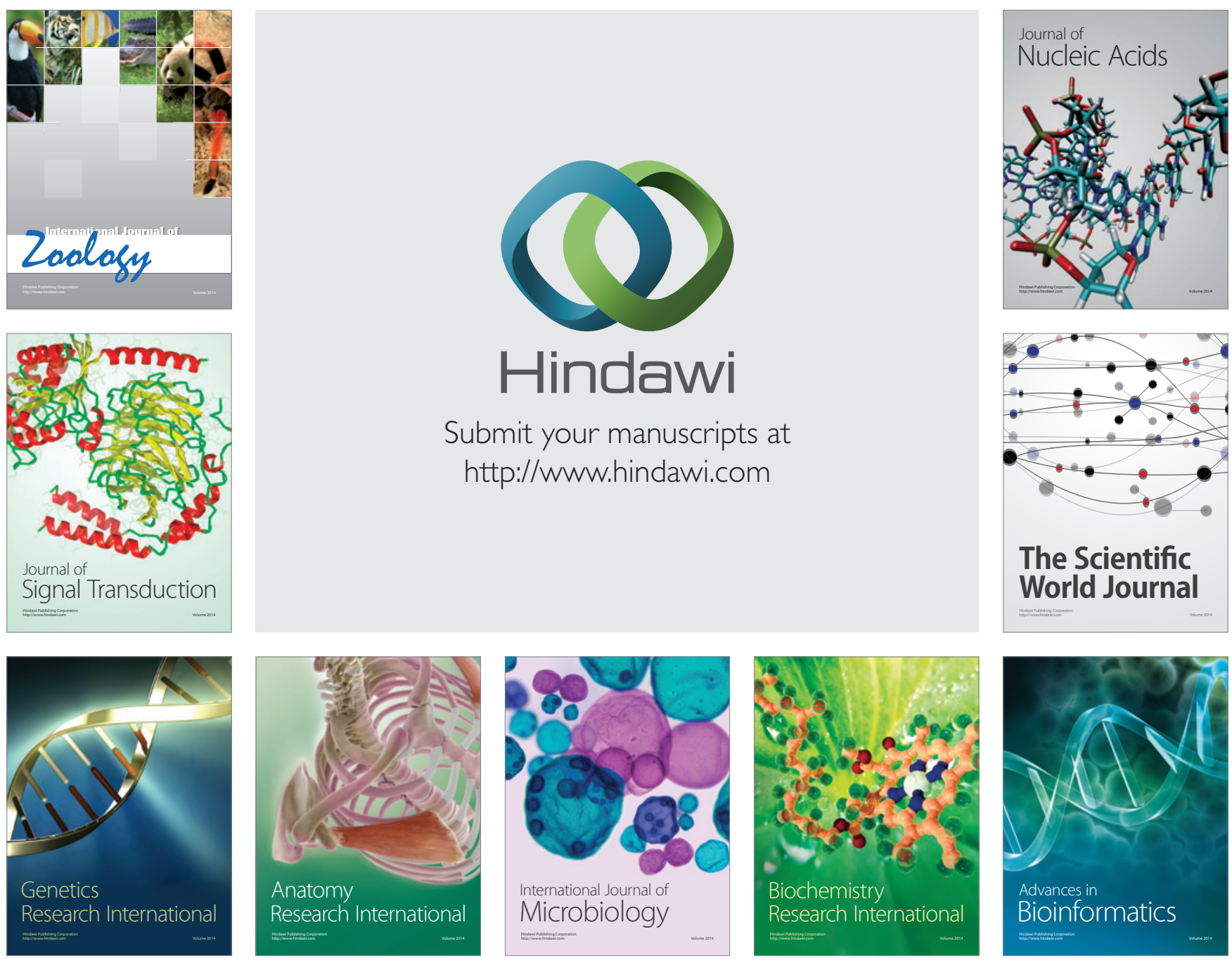

The Scientific World Journal
